\title{
Low Complexity Partial SLM Technique for PAPR Reduction in OFDM Transmitters
}

\author{
Ibrahim Mohammad Hussain \\ Department of Computer Engineering, Sir Syed University of Engineering and Technology, \\ Karachi, Pakistan \\ ibrahimh@ssuet.edu.pk,hmmiharbi@yahoo.com
}

\begin{abstract}
In wireless OFDM systems, a major cause for signal degradation during transmission is high Peak to Average Power Ratio (PAPR). Selected mapping (SLM) is one efficient technique which reduces the peaks of a signal considerably. One drawback associated with this technique is its computational complexity. The computational complexity is directly related to the complexity of the IFFT block and increases as the number of sub-carriers increases. In this paper, an effort is done to reduce the computational complexity as well as to improve the PAPR performance of the conventional SLM technique. The proposed technique called Partial Selected Mapping in which the IFFT block is divided into smaller blocks and each block is used as a separate SLM block. Partial outputs of all sub-blocks are concatenated to form the final sequence. Simulation results show that the overall computational complexity of the proposed technique is lower than that of the conventional SLM technique with better PAPR performance. A reduction of around $3 \mathrm{~dB}$ in PAPR performance at CCDF of $10^{-3}$ is achieved.
\end{abstract}

Index Terms: Computational complexity, IFFT, OFDM, PAPR, SLM, Wireless communication

\section{Introduction}

Several communication systems and techniques have been used for transferring data and information reliably at high speed over wireless channel. One such technique is Orthogonal Frequency Division Multiplexing (OFDM) used for high data rate wireless transmission [1]. In OFDM, data bits are transmitted in parallel using various carriers. Although OFDM is a multicarrier technology, it is very efficacious in mitigating the effects of multipath delay spread over a wireless radio channel. However, a major drawback with OFDM is the high Peak-to-Average Power Ratio (PAPR) of the transmitted signal. The high PAPR mainly results from certain data sequences, such as those containing all zeros or all ones. Such OFDM signals with high peaks result in poor power efficiencies. Appropriate measures should be taken to tackle this problem. Otherwise, the high PAPR signals would substantially limit the usefulness of battery powered equipment such as portable wireless devices. In addition, high peaks cause problems such as inter-symbol interference (ISI) and out-of-band radiation. Hence, it is imperative to reduce these peaks in the transmission signals. This issue has been addressed by several researchers and different algorithms have been proposed. Reduction techniques include signal clipping [2], trellis shaping algorithms [3], partial transmit sequences (PTS) [4, 5] and selected mapping (SLM) [6] - [9]. In SLM technique method multiple copies are generated from a single signal that represents the same information and the one with lowest PAPR is transmitted. Although this method is very effective in reducing PAPR, on the other hand, the computational complexity of this method increases by the number of subcarriers being used.

In this paper we have proposed a method based on the conventional SLM technique which not only has a better PAPR reduction capability but also exhibits lower computational complexity. Rest of the paper is organized as follows: in section 2, a brief overview of the

Received: August $12^{\text {nd }}, 2012$. Accepted: February $7^{\text {th }}, 2013$ 
OFDM and the PAPR issue are discussed. Section 3 gives a complete view on the SLM technique. In Section 4, the proposed algorithm known as the Partial SLM is explored along with simulation results and finally, conclusions are made in Section 5.

\section{OFDM System and PAPR}

A typical OFDM system transmitter is shown in Figure. 1 and works as follows: Serial stream of bits $\left\{b_{0}, b_{1}, b_{2}, \ldots\right\}$ are encoded using an encoder such that $b_{i}=0$ or 1 ; for $i=0,1,2, \ldots$ . The serial bit stream at the output of channel encoder is fed into a serial to parallel converter block that forms an $N$ parallel stream where $N$ represents the number of subcarriers used in the OFDM system. The number of bits entering a particular branch or subcarrier depends on the mapper (i.e. the digital modulation block) which is used after the serial to parallel converter. The number of bits per subcarrier is given by $L=\log _{2} M$ where $M$ is the constellation size used by the mapper and depends on the modulation scheme being used.

For Quadrature Phase Shift Keying (QPSK) mapper the output is denoted by D $=\left\{d_{0}, d_{1}\right.$, $\left.\ldots, d_{N-1}\right\}$ such that $d_{k}$ can take one of the values from $(1,-1, j,-j)$. Note that throughout the paper, we have used the QPSK mapper.

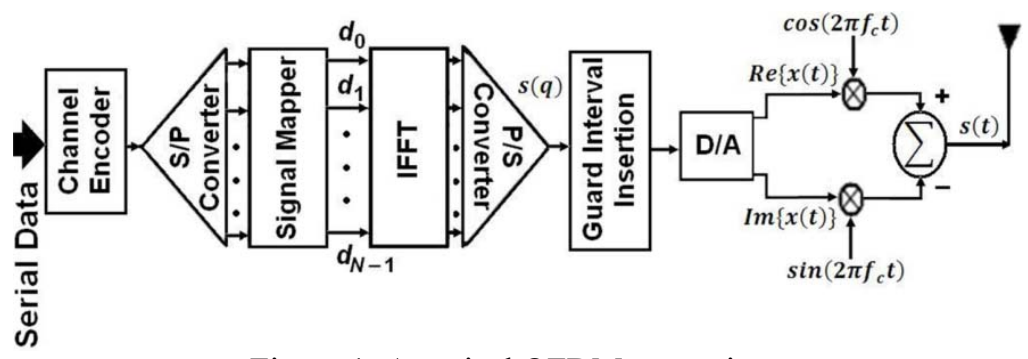

Figure 1: A typical OFDM transmitter

The Inverse Fast Fourier Transform (IFFT) block transforms the discrete complex signal into another discrete complex signal. A typical baseband signal at the output of the IFFT block is given by the following well known Inverse Discrete Fourier Transform (IDFT) equation is given as [10]:

$$
s(q)=\frac{1}{\sqrt{N}} \sum_{k=0}^{N-1} d_{k} e^{j 2 \pi k q / N} ; \quad k, q=0,1, \ldots, N-1
$$

where $s(q)$ are samples for the resultant baseband signal. The output of the IFFT block is the result of summation of various complex sinusoids with varying amplitudes and phases. At some sample points of these sinusoidal signals, constructive summations may occur, resulting in high peaks in the signal. When transmitting high peak signals through a non-linear power amplifier, distortion occurs within the transmitted signal at the output of the amplifier in the form of inter symbol interference (ISI) and out-of-band radiation. Hence, the influence of high peaks is evident at the output of a non-linear power amplifier but the point of occurrence of these peaks is at the output of an IFFT block.

One widely used measures for the power of these peaks is Peak to Average Power Ratio (PAPR) which is mathematically expressed as:

$$
P A P R=\frac{\max \{P(q)\}}{P_{\text {avg }}}=\frac{0 \leq q \leq N-1}{\frac{\max }{N} \sum_{q=0}^{N-1}|s(q)|^{\mid}}
$$


Here, $P(q)$ represents the instantaneous power and $P_{\text {avg }}$ represents the average power of the OFDM signal. Oversampling by a factor of $J$ can be used for better PAPR estimation and to capture peaks in OFDM signals. It has been shown that $J=4$ is sufficient to capture the peaks [11].

\section{Selected Mapping (SLM) Method for PAPR Reduction}

In SLM technique as shown in Figure 2, from a single OFDM sequence D having a length of $\mathrm{N}$, number of sequences are generated that represent the same information using some rotation factors and the sequence with lowest PAPR is transmitted. If the number of generated new sequences is $U$, called the SLM length, then all these sequences are the result of multiplying the incoming original OFDM sequence D by $\mathrm{U}$ different rotation factors. These factors are given in vector form as

$$
B^{(i)}=\left[b_{0}^{(i)}, b_{1}^{(i)}, \ldots, b_{N-1}^{(i)}\right.
$$

where $i=1$ to $U$ and represents the indices of these factors and $\mathrm{B}$ is the represent the rotation factor in vector form. After multiplying these factors by the original OFDM sequence $D$, we get:

$$
X^{(i)}=\left[d_{0} b_{0}^{(i)}, d_{1} b_{1}^{(i)}, \ldots, d_{N-1} b_{N-1}^{(i)}\right]
$$

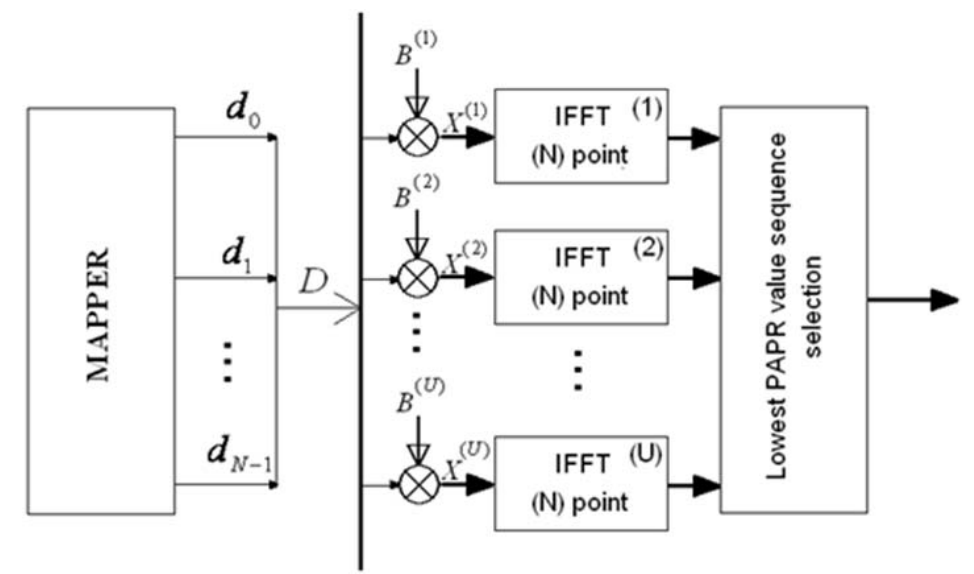

Figure 2. Conventional SLM technique at the transmitter end

The multiplication factors are phase rotations selected appropriately such that multiplying a complex number by these factors results in rotation of that complex number to another complex number representing a different point in the constellation. Hence,

$$
b_{n}^{i}=e^{-j \theta_{n}^{i}} \text { where } \theta_{n}^{(i)} \in[0,2 \pi)
$$

where $\theta$ is the angle of rotation. The rotation vectors are used as side information which are transmitted for signal recovery.

The efficiency of SLM approach depends on the amount of scrambling done by these rotation factors on the original OFDM sequence and the length of SLM $U$. As we increase number of SLM sequences, PAPR performance becomes better but at the expense of increase in system complexity.

Although SLM technique has moderate implementation complexity, this complexity increases as $U$ increases. For this reason, many researchers devoted their work in this field towards improving the complexity computation of conventional SLM $[7,8]$. 
The complexity of a typical SLM method considering no oversampling (i.e. $J=1$ ) in terms of complexity additions are:

$$
\text { Complexity }=\mathrm{UN} \log _{2} \mathrm{~N}
$$

\section{The Proposed Partial SLM Method}

In this section, a simple method has been proposed based on the SLM technique called Partial SLM. This method has better PAPR reduction performance than the conventional SLM technique with lower computational complexity. The proposed scheme is shown in Figure. 3 and works as follows: the incoming OFDM sequence $D$ is divided into $G$ number of sub sequences each of length $\hat{N}$. Each sub-sequence is fed into an IFFT block of length $\hat{N}$ and the conventional SLM is applied in each sub-block. Hence Partial SLM is nothing but the conventional SLM but now it is applied in individual sub-block. Precisely, the SLM will be applied $G$ times and for each sub-block, $U$ number of sequences are generated from a single sub-sequence and the one with the lowest PAPR (partially) is selected and stored. The procedure repeats for each sub-block till all sub-blocks are covered. Finally, all partially selected sequences are concatenated to form the final new sequence of length $N$ to be transmitted. Hence instead of using different IFFT blocks of length $N$ each (i.e. one for each SLM sequence), $G$ number of partial SLM blocks is created each of length $\hat{N}$. This is where the complexity is reduced as now we have smaller length IFFT sequences. Furthermore, the selection of $G$ is done under the following constraint:

$$
N=2^{r}, G=2^{p} \text { and } \hat{N}=\frac{N}{G},
$$

Such that $r, p$ are integers and $r>p$ with $p \neq 0$

The input to each IFFT sub-block in a particular Partial SLM block is $\hat{N}$ complex numbers from the original sequence $D$ of length $N$ such that:

$$
D=\left[d_{g}, d_{g+1}, d_{g+2}, \ldots, d_{g+\hat{N-1}}\right] \text { and } g=\hat{N}(v-1)
$$

where $v=1,2, \ldots, G$ is the sub block number. The phase rotation factors in each sub-block have a new length and they are given as

$$
\hat{B}^{(v, a)}=\left[b_{0}^{(v, a)}, b_{1}^{(v, a)}, b_{2}^{(v, a)}, \ldots, b_{\hat{N}-1}^{(v, a)}\right.
$$

where ' $a$ ' simply indicates the index of the individual rotation factors which ranges from 1 to $U$. It is very obvious that the number of rotation factors used in each sub-block in the Partial STM method remains the same (i.e. $U$ ) but the length of each rotation factor is $\hat{N}$. For instance, let us assume the following parameters: $N=8, G=2$ and $U=4$. Hence $\hat{N}=4, v=$ 1,2 and $a=1,2,3,4$. Assuming the input data is given as:

$$
D=\underbrace{\left[\begin{array}{cccccccc}
d_{0} & d_{1} & d_{2} & d_{3} & d_{4} & d_{5} & d_{6} & d_{7} \\
1 & j & j & j & -1 & -j & 1 & -1
\end{array}\right]}_{D_{1}}
$$




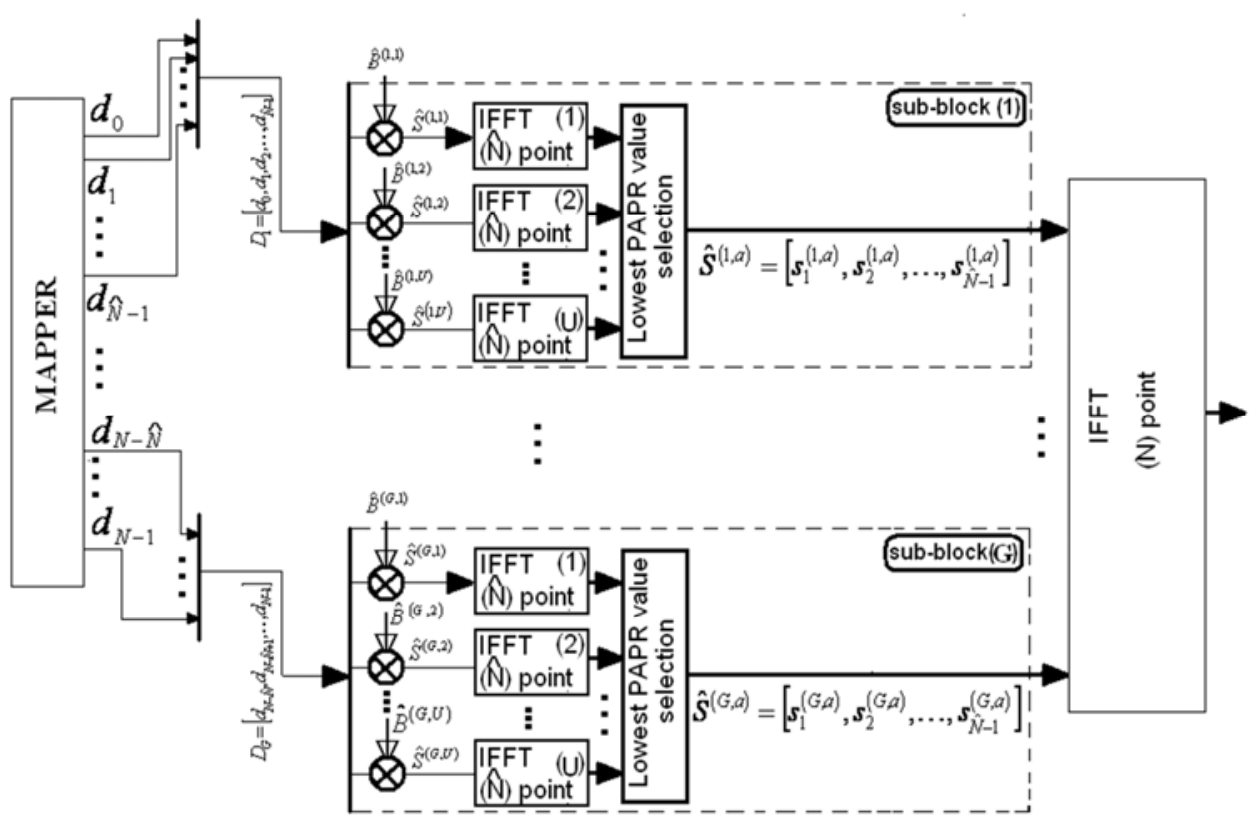

Figure 3. A block diagram of the proposed Partial SLM technique at the transmitter end

where $D_{1}$ and $D_{2}$ indicates the two partial sequences used in sub-blocks 1 and 2 respectively. If the four rotation vectors $B^{(1)}, B^{(2)}, B^{(3)}, B^{(4)}$ used in the conventional SLM are respectively given as:

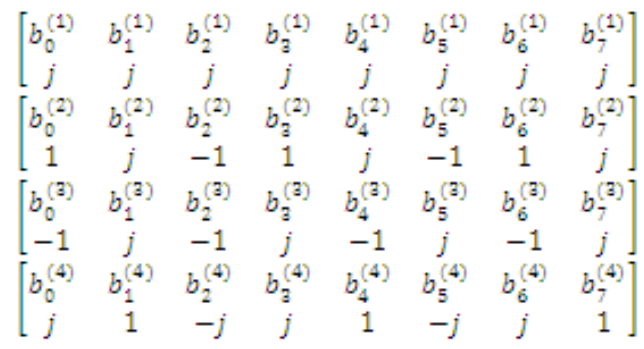

then the four rotation vectors used in each sub-block in the Partial SLM method indicated as $\hat{B}^{(v, 1)}, \hat{B}^{(v, 2)}, \hat{B}^{(v, 3)}, \hat{B}^{(v, 4)}$ for any sub-block $v$ are extracted from $B^{(1)}, B^{(2)}, B^{(3)}, B^{(4)}$ in the following manner respectively shown below such that these new rotation factors are same for all sub-blocks (i.e. $v=1,2)$ :

$$
\begin{aligned}
& {\left[\begin{array}{cccc}
b_{0}^{(v, 1)} & b_{1}^{(v, 1)} & b_{2}^{(v, 1)} & b_{3}^{(v, 1)} \\
j & j & j & j
\end{array}\right]\left[\begin{array}{cccc}
b_{0}^{(v, 2)} & b_{1}^{(v, 2)} & b_{2}^{(v, 2)} & b_{3}^{(v, 2)} \\
1 & j & -1 & 1
\end{array}\right]} \\
& {\left[\begin{array}{cccc}
b_{0}^{(v, 3)} & b_{1}^{(v, 3)} & b_{2}^{(v, 3)} & b_{3}^{(v, 3)} \\
-1 & j & -1 & j
\end{array}\right]\left[\begin{array}{cccc}
b_{0}^{(v, 4)} & b_{1}^{(v, 4)} & b_{2}^{(v, 4)} & b_{3}^{(v, 4)} \\
j & 1 & -j & j
\end{array}\right]}
\end{aligned}
$$

Now if the product of either $D_{1}$ or $D_{2}$ with one of the new rotation factors is indicated as $\hat{N}$ ${ }^{(v, a)}$ then for instance, the output of the third IFFT block in the first sub-block is given as:

$$
\overbrace{\left[\begin{array}{llllll}
1 & j & j & j
\end{array}\right] \times \overbrace{\left[\begin{array}{lllll}
-1 & j & -1 & j
\end{array}\right]}^{D_{S_{1}^{(1,8)}}}}^{\hat{B}^{(1,8)}}=
$$


To evaluate the performance of the proposed method, simulations are carried out on a 32 sub-carrier OFDM system with QPSK mapper and by generating around 10,000 OFDM symbols randomly. Figure 4 shows the Complementary Cumulative Distribution Function (CCDF) curves for the normal SLM and the proposed Partial SLM with different number of sub-block divisions $G$. In this simulation, four rotation factors are used. As it is evident from Figure. 4, conventional SLM resulted in a reduction of around of around 1.5dB at CCDF of 0.001 using only four rotation vectors. Now when Partial SLM is applied using only two subblocks, a considerable reduction in PAPR is achieved of almost $3 \mathrm{~dB}$. This is a remarkable reduction compared to the conventional SLM method which clearly shows that our proposed method out performs the conventional SLM technique.

As the number of division factor increases, the performance in PAPR reduction degrades but still it is better than the conventional SLM. Infact, when $G=8$, the reduction is almost same as it is the case in conventional SLM. When the division factor is at its maximum i.e. $G=$ 16 , the performance degrades considerably and the PAPR reduction is only $0.3 d B$. Hence, for higher division factor, Partial SLM it is not recommended for PAPR reduction as the reduction in PAPR is not substantial. This is due to the fact that the rotation factor when multiplied by smaller sequences does not scramble the overall sequence and hence produces smaller reduction in PAPR. Since smaller IFFT blocks are used, the computational complexity in terms of complex additions of the proposed method becomes

$$
\text { Complexity }=U \operatorname{UNog}_{2} \hat{N}
$$

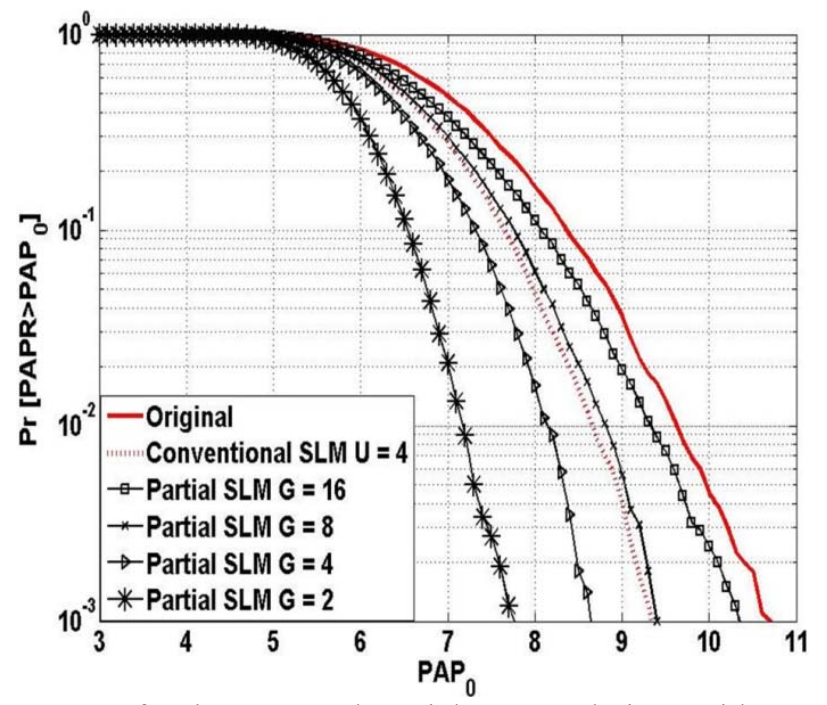

Figure 4. CCDF curves for the proposed Partial SLM technique with 32 subcarriers and different division factors

Another aspect which needs to be discussed here is the sideband spectrum of the transmitted signal using conventional SLM technique and the proposed partial SLM technique. Figure 5 compares the original spectrum of the conventional SLM sequences with the new transmitted partial SLM sequences. The Figure shows that there is no spectrum broadening and hence no distortion at the sidebands. In fact, the side spectrums for partial SLM sequences have almost $2 \mathrm{~dB}$ less power than the conventional SLM sequences which in turn show the efficiency of the proposed method in terms of power utilization especially for portable devices operated by batteries. This will result in bandwidth efficiency and reduced ISI interference with sidebands of neighboring sequences. 


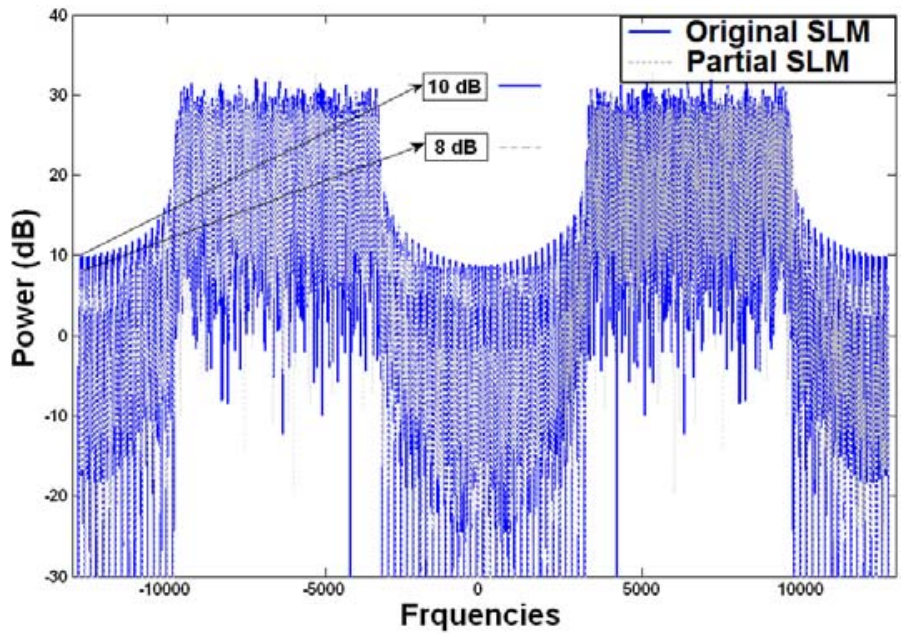

Figure 5. Power spectrum of the proposed Partial SLM technique and the conventional SLM technique with 32 subcarriers and $G=8$

Now since the complexity of the proposed system is minimized compared to the complexity of the conventional SLM technique, it would be interesting to compare these results with the work being proposed by authors in $[7,8]$. The authors in both papers developed two methods for reducing the computational complexity of the IFFT block based on some matrix conversion technique. The phase rotation vectors used in the modified SLM technique are updated using conversion matrices with variable periods. As claimed by the authors such candidate signals produced by these conversions will have little correlation and can be used to replace the IFFT blocks in the conventional SLM method. Figure 6 shows the first method for SLM modification scheme proposed by authors. The term $G$ in the Figure denoted the rotation factor. Now the number of complex additions for the proposed system is given as:

$$
\text { Complexity }=N \log _{2} N+3(U-1) N
$$

In a similar manner in the second proposed system by authors in $[7,8]$, half portion of this method is same as first one but the other part of it uses a randomly-generated phase rotation vector and an additional IFFT block to create another set of candidate signals, which are also produced by the proposed conversion matrices. Figure 7 shows the second proposed method in which the upper part is the same as the first proposed scheme, but at the lower part is changed. The computational complexity of the second proposed SLM scheme is significantly reduced and it is given as

$$
\text { Complexity }=2 N \log _{2} N+3(U-2) N
$$

Finally to complete this topic Table 1 shows the computational complexity of the partial SLM technique for different values of $U$ and $N$. The complexity reduction is more evident from higher values of $U$ and $N$. Also not that the allowed values of $G$ should not exceed the value of $\mathrm{N}$ as when $G=N$ then it becomes a conventional SLM technique. 


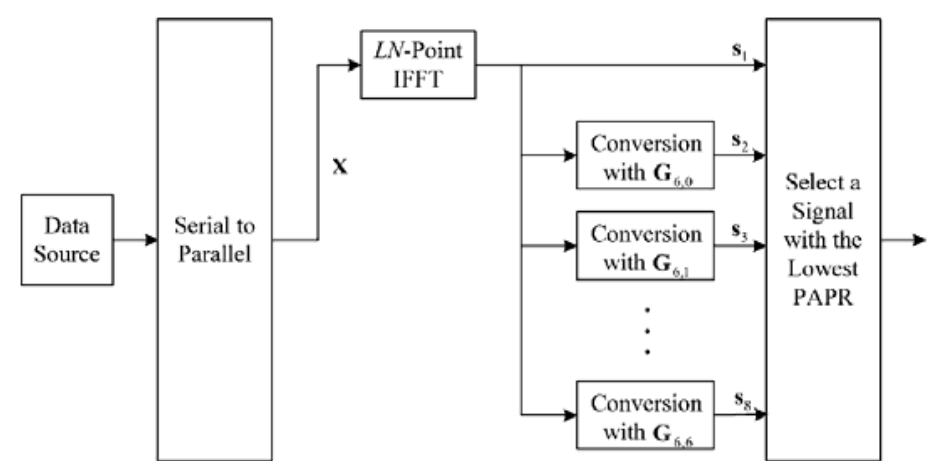

Figure 6. The first proposed method for PAPR reduction using modified SLM technique based on matrix conversion by authors in $[7,8]$

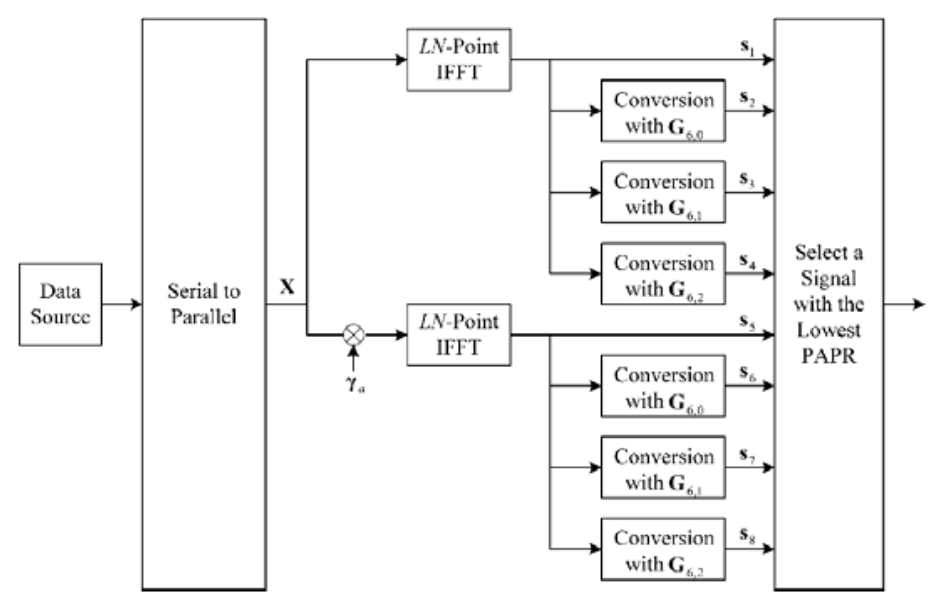

Figure 7. The second proposed method for PAPR reduction using modified SLM technique based on matrix conversion by authors in $[7,8]$

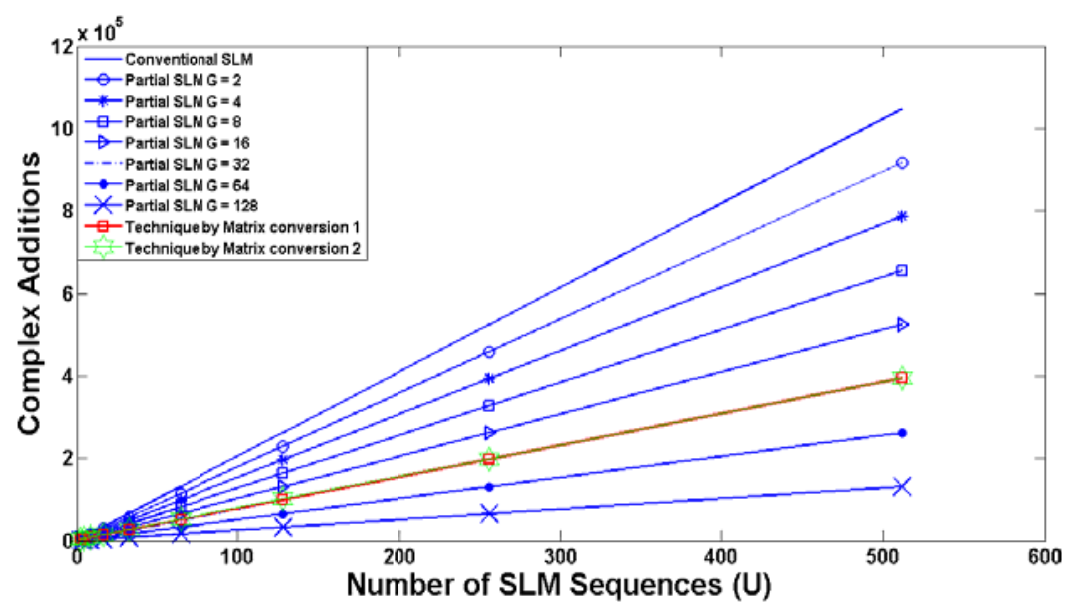

Figure 8. Complexity comparison of various techniques with the proposed partial SLM with $N$ $=256$ and different values of $U$ 
Table 1. Complexity values of the proposed partial SLM with different values of SLM sequences and subcarriers

\begin{tabular}{|c|c|c|c|c|c|c|c|c|c|}
\hline & \multicolumn{9}{|l|}{$N=16$} \\
\hline & $U=2$ & $U=4$ & $U=8$ & $U=16$ & $U=32$ & $U=64$ & $U=128$ & $U=256$ & $U=512$ \\
\hline$G=2$ & 96 & 192 & 384 & 768 & 1536 & 3072 & 6144 & 12288 & 24576 \\
\hline$G=4$ & 64 & 128 & 256 & 512 & 1024 & 2048 & 4096 & 8192 & 16384 \\
\hline \multirow[t]{3}{*}{$G=8$} & 32 & 64 & 128 & 256 & 512 & 1024 & 2048 & 4096 & 8192 \\
\hline & \multicolumn{9}{|l|}{$N=32$} \\
\hline & $U=2$ & $U=4$ & $U=8$ & $U=16$ & $U=32$ & $U=64$ & $U=128$ & $U=256$ & $U=512$ \\
\hline$G=2$ & 256 & 512 & 1024 & 2048 & 4096 & 8192 & 16384 & 32768 & 65536 \\
\hline$G=4$ & 192 & 384 & 768 & 1536 & 3072 & 6144 & 12288 & 24576 & 49152 \\
\hline$G=8$ & 128 & 256 & 512 & 1024 & 2048 & 4096 & 8192 & 16384 & 32768 \\
\hline \multirow[t]{3}{*}{$G=16$} & 64 & 128 & 256 & 512 & 1024 & 2048 & 4096 & 8192 & 16384 \\
\hline & \multicolumn{9}{|l|}{$N=64$} \\
\hline & $U=2$ & $U=4$ & $U=8$ & $U=16$ & $U=32$ & $U=64$ & $U=128$ & $U=256$ & $U=512$ \\
\hline$G=2$ & 640 & 1280 & 2560 & 5120 & 10240 & 20480 & 40960 & 81920 & 163840 \\
\hline$G=4$ & 512 & 1024 & 2048 & 4096 & 8192 & 16384 & 32768 & 65536 & 131072 \\
\hline$G=8$ & 384 & 768 & 1536 & 3072 & 6144 & 12288 & 24576 & 49152 & 98304 \\
\hline$G=16$ & 256 & 512 & 1024 & 2048 & 4096 & 8192 & 16384 & 32768 & 65536 \\
\hline \multirow[t]{3}{*}{$G=32$} & 128 & 256 & 512 & 1024 & 2048 & 4096 & 8192 & 16384 & 32768 \\
\hline & \multicolumn{9}{|c|}{$N=128$} \\
\hline & $U=2$ & $U=4$ & $U=8$ & $U=16$ & $U=32$ & $U=64$ & $U=128$ & $U=256$ & $U=512$ \\
\hline$G=2$ & 1536 & 3072 & 6144 & 12288 & 24576 & 49152 & 98304 & 196608 & 393216 \\
\hline$G=4$ & 1280 & 2560 & 5120 & 10240 & 20480 & 40960 & 81920 & 163840 & 327680 \\
\hline$G=8$ & 1024 & 2048 & 4096 & 8192 & 16384 & 32768 & 65536 & 131072 & 262144 \\
\hline$G=16$ & 768 & 1536 & 3072 & 6144 & 12288 & 24576 & 49152 & 98304 & 196608 \\
\hline$G=32$ & 512 & 1024 & 2048 & 4096 & 8192 & 16384 & 32768 & 65536 & 131072 \\
\hline \multirow[t]{3}{*}{$G=64$} & 256 & 512 & 1024 & 2048 & 4096 & 8192 & 16384 & 32768 & 65536 \\
\hline & \multicolumn{9}{|c|}{$N=256$} \\
\hline & $U=2$ & $U=4$ & $U=8$ & $U=16$ & $U=32$ & $U=64$ & $U=128$ & $U=256$ & $U=512$ \\
\hline$G=2$ & 3584 & 7168 & 14336 & 28672 & 57344 & 114688 & 229376 & 458752 & 917504 \\
\hline$G=4$ & 3072 & 6144 & 12288 & 24576 & 49152 & 98304 & 196608 & 393216 & 786432 \\
\hline$G=8$ & 2560 & 5120 & 10240 & 20480 & 40960 & 81920 & 163840 & 327680 & 655360 \\
\hline$G=16$ & 2048 & 4096 & 8192 & 16384 & 32768 & 65536 & 131072 & 262144 & 524288 \\
\hline$G=32$ & 1536 & 3072 & 6144 & 12288 & 24576 & 49152 & 98304 & 196608 & 393216 \\
\hline$G=64$ & 1024 & 2048 & 4096 & 8192 & 16384 & 32768 & 65536 & 131072 & 262144 \\
\hline \multirow[t]{3}{*}{$G=128$} & 512 & 1024 & 2048 & 4096 & 8192 & 16384 & 32768 & 65536 & 131072 \\
\hline & \multicolumn{9}{|l|}{$N=512$} \\
\hline & $U=2$ & $U=4$ & $U=8$ & $U=16$ & $U=32$ & $U=64$ & $U=128$ & $U=256$ & $U=512$ \\
\hline$G=2$ & 8192 & 16384 & 32768 & 65536 & 131072 & 262144 & 524288 & 1048576 & 2097152 \\
\hline$G=4$ & 7168 & 14336 & 28672 & 57344 & 114688 & 229376 & 458752 & 917504 & 1835008 \\
\hline$G=8$ & 6144 & 12288 & 24576 & 49152 & 98304 & 196608 & 393216 & 786432 & 1572864 \\
\hline$G=16$ & 5120 & 10240 & 20480 & 40960 & 81920 & 163840 & 327680 & 655360 & 1310720 \\
\hline$G=32$ & 4096 & 8192 & 16384 & 32768 & 65536 & 131072 & 262144 & 524288 & 1048576 \\
\hline$G=64$ & 3072 & 6144 & 12288 & 24576 & 49152 & 98304 & 196608 & 393216 & 786432 \\
\hline$G=128$ & 2048 & 4096 & 8192 & 16384 & 32768 & 65536 & 131072 & 262144 & 524288 \\
\hline$G=256$ & 1024 & 2048 & 4096 & 8192 & 16384 & 32768 & 65536 & 131072 & 262144 \\
\hline
\end{tabular}

Figure 8 show a comparison of computational complexities of the work been done by authors in [7,8] and the proposed partial SLM method. The results are generated for fixed value of $N=256$ and varying the values of $U$. Note that the proposed partial SLM technique out performs the conventional SLM technique in terms of number of complex additions. For instance, for $U=512$ SLM sequences, the number of complex additions required by the conventional SLM technique is around more than 1,000,000 additions where is for partial SLM technique with $G=2$ requires below 900,000 complex additions. Furthermore as $G$ increases the complexity of partial SLM technique reduces considerably especially for $G=128$ which is 
around 100,000 only which is more than 10 times less than the conventional SLM technique. Now comparing the complexity result with those techniques propose by authors in $[7,8]$, it is obvious that the complexity of both the techniques is less than the proposed partial SLM technique till $G=16$. For $G=32$ the performance of the techniques proposed by the authors and the partial SLM technique is almost same. Beyond $G=32$ (i.e. $G=64,128$ ) the computational complexity is better than both the proposed techniques by the authors. This does not only show the efficacy of the partial SLM technique in terms of PAPR reduction capability but also its reduced complexity compared to the conventional and some other well renowned techniques being proposed in the past.

\section{Conclusions}

SLM technique is a very efficient technique for reducing PAPR. A major disadvantage of this technique is its implementation complexity as the length of SLM and number of subcarriers increase. To handle this problem, a novel technique based on dividing the transmitted sequence into sub sequences and applying the SLM algorithm individually is proposed. Simulation results show that the proposed method outperforms the conventional SLM technique both in terms of PAPR reduction and computational complexity. In fact, the best performance in terms of PAPR occurs when the number of division factor is two with complexity reduction by a factor of approximately 1.2. Furthermore, the complexity of the proposed technique is less than that of some well established technique for PAPR reduction in OFDM systems.

\section{References}

[1] Special Issue on 4G Mobile Comm.: Toward Open Wireless Architecture. IEEE Wireless Comm. 2004, vol. 11, no. 2, pp. 4-6, 2004.

[2] Kaiyuan Xue, Hongwen Yang and Shenglan Su, "The Clipping Noise and PAPR in the OFDM System”, in proc. Of the WRI International Conference on Communications and Mobile Computing 2009, vol. 1, pp. 265 - 269, 2009.

[3] Hideki Ochiai, "A novel trellis-shaping design with both peak and average power reduction for OFDM systems", IEEE Transactions on Comm., vol. 52, no. 11, pp. 1916 1926, 2004.

[4] Cimini L.J., Jr. Sollenberger N.R., "Peak-to-average power ratio reduction of an OFDM signal using partial transmit sequences", IEEE Communications Letters, vol. 4, no. 3, pp. 86 - 88, 2000.

[5] Yang Zhang, Qiang Ni, Hsiao-Hwa Chen, "A new partial transmit sequence scheme using genetic algorithm for peak-to-average power ratio reduction in a multi-carrier code division multiple access wireless system", International Journal of Autonomous and Adaptive Communications Systems, vol. 2, no. 1, pp. 40-57, 2009.

[6] Jayalath, A.D.S., Tellambura C., "SLM and PTS peak-power reduction of OFDM signals without side information", IEEE Transactions on Wireless Communications, vol. 4, no. 5, pp. $2006-2013,2005$.

[7] Chin-Liang Wang, Sheng-Ju Ku., "Novel conversion matrices for simplifying the IFFT computation of an SLM-based PAPR reduction scheme for OFDM systems", IEEE Transaction on Communication, vol. 57, no. 7, pp. 1903 - 1907, 2009.

[8] Chin-Liang Wang, Yuan Ouyang, "Low-complexity selected mapping schemes for peakto-average power ratio reduction in OFDM systems", IEEE Trans. on Signal Processing, vol. 53, no. 12, pp. 4652 - 4660, 2005.

[9] Masato Saito, Akihiro Okuda, Minoru Okada, Heiichi Yamamoto, "Peak-to-average power ratio reduction of multi-carrier CDMA signals with interleaver-based selected mapping", European Transactions on Telecommunication, vol. 17, no. 6, pp. 695 - 701, 2006.

[10] Alan V. Oppenheim, Ronald W. Schafer, "Discrete-time signal processing",. Prentice Hall: Englewood Cliffs, 1989. 
[11] C. Tellambura, "Use of m-sequences for OFDM peak- to-average power ratio reduction", IEE Elec. Lett., vol. 33, no. 15, pp. 1300-1301, 1997.

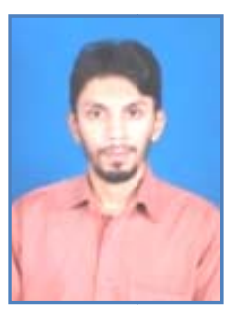

Ibrahim Mohammad Hussain was born in Pakistan in 1979. He obtained BSc and MSc from University of Engineering and technology, Karachi , Pakistan in 2002 and 2007 respectively. Currently, he is an assistant professor at Department of Computer Engineering, Sir Syed University of Engineering and Technology, Pakistan. His research interests are digital image processing, computer vision techniques and wireless communication systems. He has published more than 20 international conference and journal papers. Mr. Ibrahim Hussain can be reached at Ibrahim@ssuet.edu.pk 